Amongst these are what appear to be absorption lines, but, judging from other regions of the spectrum, the bright lines are the authentic ones, and the apparent absorption lines are more likely to be interspaces between bright lines, and have little or no significance as spectrum lines.

The well-known series of bright nova lines on the less refrangible side of $\mathrm{H} \beta$ at $\lambda \lambda_{4924}, 5018,5 \mathrm{I} 69$, 5276,5317 , seen in Nova Persei and Nova Aurigæ, are not well shown in the Madrid spectra, only the first two of these being faintly seen. This is probably due to the plates used by Iñiguez being not very sensitive to this part of the spectrum, and not due to any real lack of lines in the spectrum.

These bright lines and those previously mentioned between $\mathrm{H} \gamma$ and $\mathrm{H} \delta\left(\lambda \lambda_{4} \mathrm{r} ; 6,4233,4300\right)$ were recorded by Sir Norman Lockyer in a series of Royal Society papers on Nova Persei in 1902, as being due to the enhanced lines of iron $\lambda \lambda\left\{\begin{array}{l}41735 \\ 4179^{\circ} 0\end{array}\right\}, 4233^{\circ} 3,\left\{\begin{array}{l}4296^{\circ} 7 \\ 4303.3\end{array}\right\}$, $4924^{\circ} x, 5018^{\circ} 6,5169^{\circ} 2,5276 \%$, 5316\%. These are the only enhanced lines of iron in the two regions mentioned, and they are all represented by strongly marked lines in a Crgni. At direct comparison of the $\alpha$ Cygni spectrum with that of Nova Persei will show that these isolated strong lines of a Cygni fall exactly on the middles of the broad, bright nova lines.

Some of the lines mentioned have, in previous publications on novæe spectra, been ascribed to various origins. The $\lambda_{4924}$ and $\lambda_{5}$ or 8 lines have often been referred to as helium lines, although much stronger lines of the same element have been lacking. 'The line $\lambda_{5}$ or 8 is also sometimes identified with the chief nebular line. The line $\lambda_{5} \mathrm{I} 69$ is often referred to as being probably the " $b$ " group of magnesium, and the line $\lambda 5316^{\circ} 9$ as being probably the chief corona line. The fact that all these lines occur together as strong lines in the spectrum of a normal star- $\alpha$ Cygni -and that they can all be atequately accounted for by specially behaved lines of one chemical elementand those the only special lines of that element in the region discussed-must surely be taken as convincing evidence that the identity is a real one.

In the region between $\mathrm{H} \gamma$ and $H \beta$, the nova spectrum is far more complex, but so also is that of a Cygni, and here again the chief lines in the nova spectrum agree in position with lines or groups of lines in a Cygni. In this part of the a Cygni spectrum there are enhanced lines of iron, magnesium, chromium, and titanium, but there is little or no doubt that in the nova spectrum the chief lines, other than those of hydrogen and calcium, are due to iron.

The most striking changes between the spectrum of March io and that of March 22 are: (I) the occurrence of what seems to be a fine bright reversal in the middles of the dark $\mathrm{H} \gamma$ and $\mathrm{H} \delta$ bands (more particularly the former), and (2) the appearance of a bright band, the centre of which is at about $\lambda_{4440}$, superposed on what was a broad, dark band on March 19. One of the strongest lines in Wolf Rayet spectra occurs at or near this position (4442), and the two lines may possiblv be identical. Unless, however, some of the other strong Wolf Ravet bands, such as $\lambda \lambda_{4} 652$, $5692,58 \times 3$, are also found in the spectra, little weight can be attached to the suggested identification.

F. F. BАхАNDALI.

Magnitude observations of the nova are published in Nos. $4566-67$ of the Astronomische Nachrichten, and Dr. Rosenberg describes his observations of the spectrum at the Tubingen Observatory. On March ig a red-sensitive plate showed well-marked radiations corresponding to $\mathrm{H} \alpha, \mathrm{H} \beta$, and $\mathrm{H} \gamma$. Their breadth was about 30 A.U., of which 9 A.U. was shifted towards the red, and $2 \mathrm{I}$ A.U. towards the violet from the normal positions. $\mathrm{H} \gamma$ presented three maxima at $\lambda \lambda 4348,4339$, and 4332 respectively, and an examination of the spectrum for polarisation effects gave a negative result.

In a report to the Harvard College Observatory Prof. Frost states that a spectrogran taken on March $I_{5}$ shows the $\mathrm{H}$ and $\mathrm{K}$ lines bright, at about their normal positions; they are strong and broad and crossed by very sharp, dark lines. The lines at $\lambda_{4923}$ and $\lambda_{5} 016$, which Prof. Frost ascribes to helium, are strong, both bright and dark, but the line at $\lambda 44 \tau^{2}$ is not conspicuous, although probably present.

WiLliam E. RolstoN.

\section{THE LOSS OF THE "TITANIC."}

THE terrible loss of life on account of the disaster to the Titanic has directed emphatic attention to various aspects of the employment of wireless telegraphy in times of crisis at sea. The point which is at the moment attracting most of the public attention is that of the erroneous messages, or alleged messages, which appeared in the newspapers in the day or two following the disaster. Possibly some of these messages may have been invented by imaginative reporters, but others seen to have been perversions of messages which had actually passed between vessels at sea, but which were not concerned with the accident. This kind of mistake is well illustrated by the transformation undergone by a message containing the words, "Am towing oil-tank to Halifax." Such mistakes as these are possible in all kinds of telegraphy, but they probably arose in the present case at the hands of some of the amateur wireless telegraphists that swarm on the American coast. Some of these amateurs, it is widely believed, may indeed have originated of set purpose a number of the early reassuring messages, and it is clear that the possibility of rigging the insurance market by such messages affords motive enough for their concoction. It is most unlikely that intelligence of this character should have been sent in irresponsible moments by operators on liners, for the operators are under the direct control of the captains, the service discipline is strict, and every message has to be recorded.

A11 this raises more prominently than ever the chaotic condition of wireless telegraphy in the United States. For years the legritimate users of wireless telegraphy have complained of the unbounded freedom enjoyed and abused by the American amateur; perhaps they may now look forward to the imposition of some salutary restrictions. But besides that aspect of the matter just discussed, there is another which this catastrophe has brought into prominence. It is now impressed on us that the most urgent call for help will pass unheeded if none of the operators on the ships within hail are on duty. In fact, it seems to have been a mere chance that the Carpathia operator was at his apparatus at the time the Titanic called. On ships that carry only one operator-and very few carry more-the man cannot always be on the look-out. For this deadly contingency one obvious remedy is for each ship to carry more operators; another remedy lies in the provision of an apparatus that will ring up the telegraphist when a message reaches it. This latter desideratum is, unhappily, as yet unattained.

Engineering aspects of the disaster are discussed in the leading article in Engineering for April I9. As but little definite information is available as yet, the drawing of conclusions is premature, but several questions present themselves as ripe for discussion and settlement. The effect of centre-lins or longitudinal wing bulkheads is one of these Such have NO. 2217 , VOL. 89] 